\title{
Integration of Beer-Lambert and van 't Hoff Laws for Salinity Detection using Remote Sensing Spectroscopy
}

\author{
A. DeHni ${ }^{a, *}$ AND N. KhelOUfi ${ }^{b}$ \\ ${ }^{a}$ Département Système d'Information à Référence Spatial - S.I.R.S., Service Modélisation des Systèmes Spatiaux, \\ Centre des Techniques Spatiales, BP 13 rue de Palestine Arzew, 31200 Oran, Algeria \\ ${ }^{b}$ Département Géodésie Spatiale, Service Modélisation Ionosphérique par GNSS, \\ Centre des Techniques Spatiales, BP 13 rue de Palestine Arzew, 31200 Oran, Algeria
}

\begin{abstract}
This paper presents a technique based on optical detection of the salinity by integrating both Beer-Lambert and van 't Hoff laws regarding remote sensing spectroscopy. Classical detection techniques using polychromatic waveband by field computation and experimental data are, on the one hand, time-consuming and on the other hand expensive and less accurate. Therefore, best precision is reached by implementing monochromatic wavelength from multispectral bands (Landsat 8) and spectroscopy USGS database. Our method aims to identify spectral reflectance of $\mathrm{NaCl}$ and $\mathrm{KCl}$ salts through satellite images using calibration process and atmospheric correction by dark object subtraction method, thermal infra-red sensor, and operational land imager. We have performed an automated tool detection using intrinsic physical parameters such as absorbance, radiance, reflectance, and transmittance, related dynamically to osmotic pressure, thermodynamic temperature from surface emissivity, and finally molar concentration of salts. The results illustrate that osmotic pressure is influenced by temperature at infra-red shortwave and more sensitive for thermal infra-red sensor, blue, and costal bands, which is affected by aerosol scattering diffusion and water absorption attenuation. This means that pressure and low temperature act synergistically in mixture mass attenuation coefficient or quantitative concentrations of materials and molar absorption. The infra-red short-wave properties are the most diffusing according to sigmoidal model adaptation. The best correlation was demonstrated by vapor pressure (exponential model) for the blue wave band (99.43\%) and the dose response model (DR-Hill) for panchromatic wave band (98.4\%). The results confirm that for small particles, the Rayleigh diffusion is predominate depending on the concentration of electrolyte and the wavelength polarization. The models demonstrate that the wave bands near 260, 1400, and $2100 \mathrm{~nm}$ are more sensitive to humidity and salinity absorption. Spectral curves of halite and sylvite salts have more intense electronic and vibrational effects in the short-wave field according to their solubility product chromophores, such as water, due to the presence of salt.
\end{abstract}

DOI: 10.12693/APhysPolA.136.367

PACS/topics: optical detection, salinity absorbance, Beer-Lambert, van 't Hoff, monochromatic, Landsat 8

\section{Introduction}

In this paper we investigated a tool by detection and identification for salts by using multiple spectroscopies such as visible, near-infrared, and thermal spectroscopy as a fast and inexpensive approach to the salinity characterization. Remote sensing is a technology that gives more spectral information for salinization monitoring. The optical signal is directly influenced from the Fresnel reflectance effects, and decrease with soil depth from the Fraunhofer diffraction effect. In the past, approaches have been attempted to discriminate soil properties, absorption, and reflectance process. Spectral reflectance in the multispectral field of Landsat 8 offers a nondestructive technique for electrolyte salinity detection by discrimination for carbonate / silicate minerals.

The utility of multispectral remote sensing techniques for discriminating salinity (halite, sylvite) is based on the solubility product differences among their spectral properties. Prediction of soil salinity by using

*corresponding author; e-mail: adehni@cts.asal.dz spectroscopy is a new challenge that can be used in realtime to the specific detection applications that are time consuming to set up salinity monitoring. Research is documented regarding the subtle changes in physical absorption of some salts until spectroscopy have been extracted potentially. Different effects were discussed, such as linearity concept related to the wavelength, the absorbance issued by the Beer-Lambert and van 't Hoff laws, noise effects, and sensor calibration (bandpass wide, signal to noise, constructive wavelength cycles).

Some physical parameters such as attenuated total reflection (ATR), the infrared absorption coefficient in a pure component unmeasurable for different water level because the Beer-Lambert law assumes the additivity linearity and diffuse reflection which have become popular are increasingly being adopted for quantitative transmission analysis of solid materials and mixture in midinfrared spectroscopy [1]. Hence, the differential spectral curve fitting from the Fourier transform infrared (FT-IR) spectra of the biomaterial tested at different water levels from every pulverization times knowing biomaterial weights, and the absorbance ratios were determined which were needed to compute the $r$-matrix and solve the system constraining the solution to satisfy the BeerLambert law by fitting curves in the whole spectrum [1]. 
The Beer-Lambert law is used widely in infrared spectroscopy for analyzing polymer degradation and oxidation in biological tissue and promoting the analysis of a mixture by spectrophotometry without the need for extensive pre-processing of the sample by generating the total mass attenuation coefficients or quantitative concentrations of materials related to the linear attenuation for different energy photons (from $1 \mathrm{keV}$ to $100 \mathrm{GeV}$ ) [2].

Commensurate spectral databases of mineral salts have been explored for spectroscopy salinity modelling. Spectral databases are available at USGS website [3]. Quantities of salts, moisture content (which means the Band 1 of Landsat 8), color, and surface roughness [4]. Farifteh et al. (2006) determine spectral reflectance data acquired by spectroradiometer. The radiation reflected from soil surfaces varies with changes in soil constituents and wavelengths [5]. The soil spectral variations occur in narrow wavelength that gradually fade with coarser bandwidths, or by limited number of spectral bands [6]. However, waveband sensors like Landsat, which have bad capacity for saline affected areas delineation by ultraspectral resolution.

Generally, hyperspectral remote sensing allows subtle features changes in salt spectrometry. For more comprehension, hyperspectral imager provides reflectance threshold at $10 \mathrm{~nm}$ by interval from 400 to $3500 \mathrm{~nm}$. Hyper spectral imageries have good potential quantification of salinity because the hydrogen chromophores result in subtle spectral changes [7]. Consequently, the electronic processes and photon vibrational processes of mineral salts in water forming the carbonates / calcites are determined directly for halite and sylvite salts. This is challenge for monitoring soil moisture and soil salts concentration in arid or semi-arid lands [9]. In Ref. [10] the potential and constraints of salinity identification by remote sensing techniques, including hyperspectral technology were discussed. In 1997, in Ref. [11] the authors have successfully applied a visible and near infrared analysis approach to retrieve field soil moisture and salinity separately by using the hyperspectral airborne sensor. Nawar et al. applied partial least squares regression and multivariate adaptive regression splines to calibrate soil salt concentration models. Firstly, most correlation between spectral reflectance and soil salinity are strongly adapted for severely salt-affected soils, but are weaker for slightly and moderately salt-affected areas [9]. The latter is the challenge for salinity identification [12]. Secondly, reflectance measurements are affected by soil texture [13], producing uncertainties in specific moisture or salt content assessments. Thirdly, salt and moisture component have been masked by the real reflectance. Consequently, increased moisture reduces albedo in a nonlinear manner, and reduces the absorption features of the salty minerals [14].

For reflectance correction processing, an atmospheric correction was established by dark object subtraction (DOS) method under Idrisi software where the scattering is removed by subtracting the haze value from every pixel in the waveband. This technique is effective for haze correction in multispectral data, but not used for hyperspectral data. Calculating surface temperature (LST) by Landsat 8 becomes an important factor controlling many physical, chemical, and biological processes of the Earth [15]. The DOS technique has been considered more suitable image-based method to erase the haze component caused by additive scattering from remote sensing data [16].

Following January 6, 2014, following recommendations of USGS for not using TIRS Band 11 due to its larger calibration uncertainty, only Band 10 was included in the salinity modeling using van 't Hoff and Planck's laws. We have also addressed the Beer law as the concept of absorption spectroscopy for multispectral analysis according to the stray effect in nanometer scales. Therefore, the Beer law obeys a monochromatic concept using narrow absorption from salts, a light beam received by Landsat 8 sensor with a narrower spectral bandwidth, less than $100 \mathrm{~nm}$. Polychromatic concept cannot permit a detection ray under $100 \mathrm{~nm}$ bandpass, because the conventional optical instrumentation is impossible for old satellite platform such as Landsat 5, 7 .

The problem is solved partially in the push broom design of Landsat 8 because this type of instrument uses a continuum source according to focal interferometry technology. The goal of this approach is commensurate by each simultaneous instrumental measurement and that is why a continuum source instrument is much more expensive. A monochromatic concept is deduced from discretizing the polychromatic wavebands of Landsat 8, positioned between the wavebands of each spectral charge coupled device (CCD) detector, but its function is only to isolate one line from the line source and to reduce stray light effect. The line width of the light source is typically about $0.1 \mathrm{~nm}$, not very much less than the absorption line width. Thus, adherence to the Beer law is not perfect, but its good enough at low concentrations. The disadvantage is that one needs to purchase a separate lamp for each element one intends to measure. That is why our optical physic approach uses straight light linearity validation through the Beer-Lambert and van 't Hoff laws for a better adaptation.

The main objectives of this research are to: (i) implement both the laws of Beer-Lambert and van 't Hoff for concentration and osmotic pressure determination in the Landsat images, based on salt spectroscopy (halite, sylvite) by both cubic crystal solubility and field based multispectral data; and (ii) develop an improved identification and automatic detection of spectral salts to quantify low, medium, and high concentration levels at the maximum wavelength (absorbance) across any electrolyte. The novelty in this paper is the technique by which the reflectance browsed by the salt spectroscopy is estimated in multispectral images by monochromatic formalism, based on regression fitting, radiometric and atmospheric calibration. 


\section{Methodology}

\subsection{Experimental}

In order to perform the experiment, our experimental tool remains a consistent spectral database which is used for comparison and validation by post processing detection. The first material "image" is obtained with geodetic reference: World Geodetic System (WGS84) datum. The OLI images Landsat 8 is acquired over the Oran region in Algeria for spectroscopy analysis. The OLI image is acquired on 14 May 2016 (path/row 198/35). The second material used is the currently published version of the USGS spectral library splib06a [6] and becomes the leading database for materials detection.

\subsection{Methods}

The global methodology is inspired by the experiments performed by several authors [9] which makes a flowchart for detection and identification of spectral reflectance for mineral salts. The continuum-removal techniques have been addressed by filtering the minimum variations in the spectral absorption, the bandwidth, and depth of the major peaks absorption bands. The flowchart (Fig. 1) illustrates the general methodology in remote sensing spectroscopy.

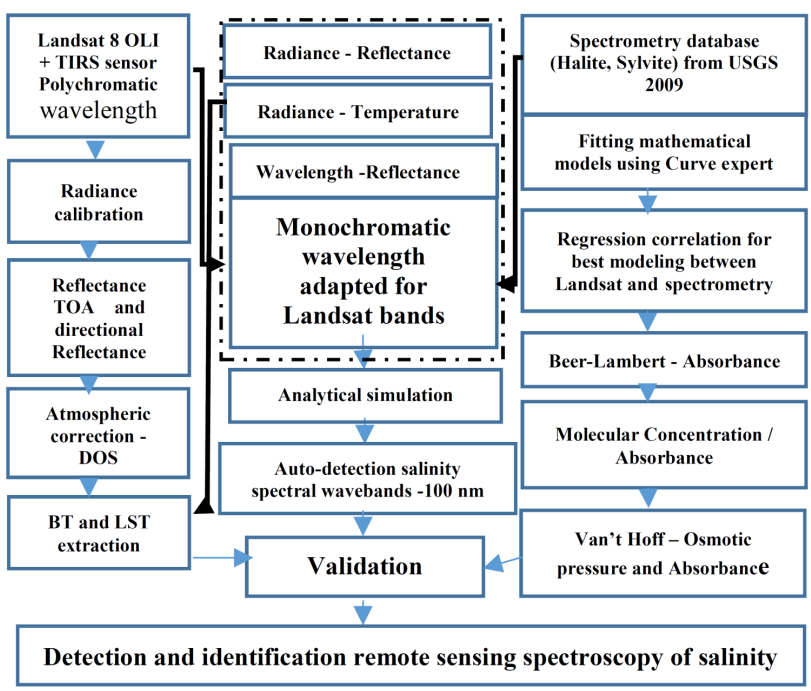

Fig. 1. Flowchart of detection and simulation of salinity by spectroscopy remote sensing techniques.

\subsubsection{Spectral attributes calculation from spectroscopy} and Landsat sensor - depth of absorption in wavebands

The absorption bands from halite salt is determined by the continuum removal technique [12] for continuum quantification in absorption depth of the Landsat bands. This method implies the calculation of the original reflectance values $\rho_{\text {original }}$ on each waveband $\lambda$ by the corresponding values projected on the stray line $\rho_{\text {continuum }}$ connecting the absorption band. The depth of an absorption band $D$ is calculated by Eq. (1) [17]:

$$
D=1-\frac{\rho_{\text {original }}}{\rho_{\text {continuum }}} .
$$

The $D$ values for absorption bands were fitted in spectral curve as a function of stray light spectra values for the USGS spectroscopy database, using the residual correlations techniques. To summarize our objective, we have explored the experimental fitting under Curve Expert software to achieve this study (Fig. 2). At number wave of $40000 \mathrm{~cm}^{-1}$ a vibrational frequency is around $5 \times 10^{14} \mathrm{~Hz}$ for $\mathrm{NaCl}$ electrolyte polarization.

\subsubsection{Absorbance and osmotic pressure from the Beer-Lambert and van 't Hoff laws}

Analytic chemistry technique was addressed by measuring intrinsic parameters related to the absorbance for bichromatic rays, which is related to the Beer-Lambert law by the formulae

$$
\begin{aligned}
A & =\log \left(P_{01}+P_{02}\right) \\
& \quad-\log \left(P_{01} \times 10^{-\varepsilon_{1} b C}+P_{02} \times 10^{-\varepsilon_{2} b C}\right),
\end{aligned}
$$

where $\varepsilon_{1}=\varepsilon_{2}$, implying

$$
\begin{aligned}
A & =\log \left(P_{01}+P_{02}\right) \\
& -\log \left(\left(P_{01}+P_{02}\right) \times 10^{-\varepsilon b C}\right), \\
A & =\log \left(P_{01}+P_{02}\right) \\
& -\log \left(P_{01}+P_{02}\right)-\log \left(10^{-\varepsilon b C}\right)=\varepsilon b C
\end{aligned}
$$

Here $P_{01}, P_{02}$ represent respectively the light energy of wavelength $\lambda_{1}$ and $\lambda_{2}, \varepsilon_{1}$ and $\varepsilon_{2}$ are coefficients of molar absorption, $b$ is the path length, and $C$ is the molar concentration.

Firstly, at constant path length, the absorbance is directly proportional to the concentration of mineral salt. Absorbance increases linearly with concentration as predicted by the Beer-Lambert law. The linearity concept will be a practical experimental question case by case, which is affected by the substance and instrumentation noise. The absorbance obeys the Beer-Lambert law in the whole concentration using a monochromatic wavelength and validation is apprehending for more dilute solutions "infinite dilution and null interactions".

Whereas, for normal calibration curve is asymptotic to the transmittance of unabsorbed light if solution concentration is important, the molecular properties are modified (the solute becomes solvent, the molecules are statistically neighbor...) and the Beer-Lambert law is not obeyed (limit detection of many spectrophometer). Secondly, the absorbance is directly proportional to the thickness or path length of absorbing material which is determined by linear fitting at low concentration. For each unique wavelength, the absorbance of the mixed solution is exactly equal to the sum of the absorbance solutions. The linear optical length is adapted by van 't Hoff law using osmotic pressure formalism. This matter is considered as a perfect match to Eq. (5) and it is commensurate for osmotic pressure for diluted solution 

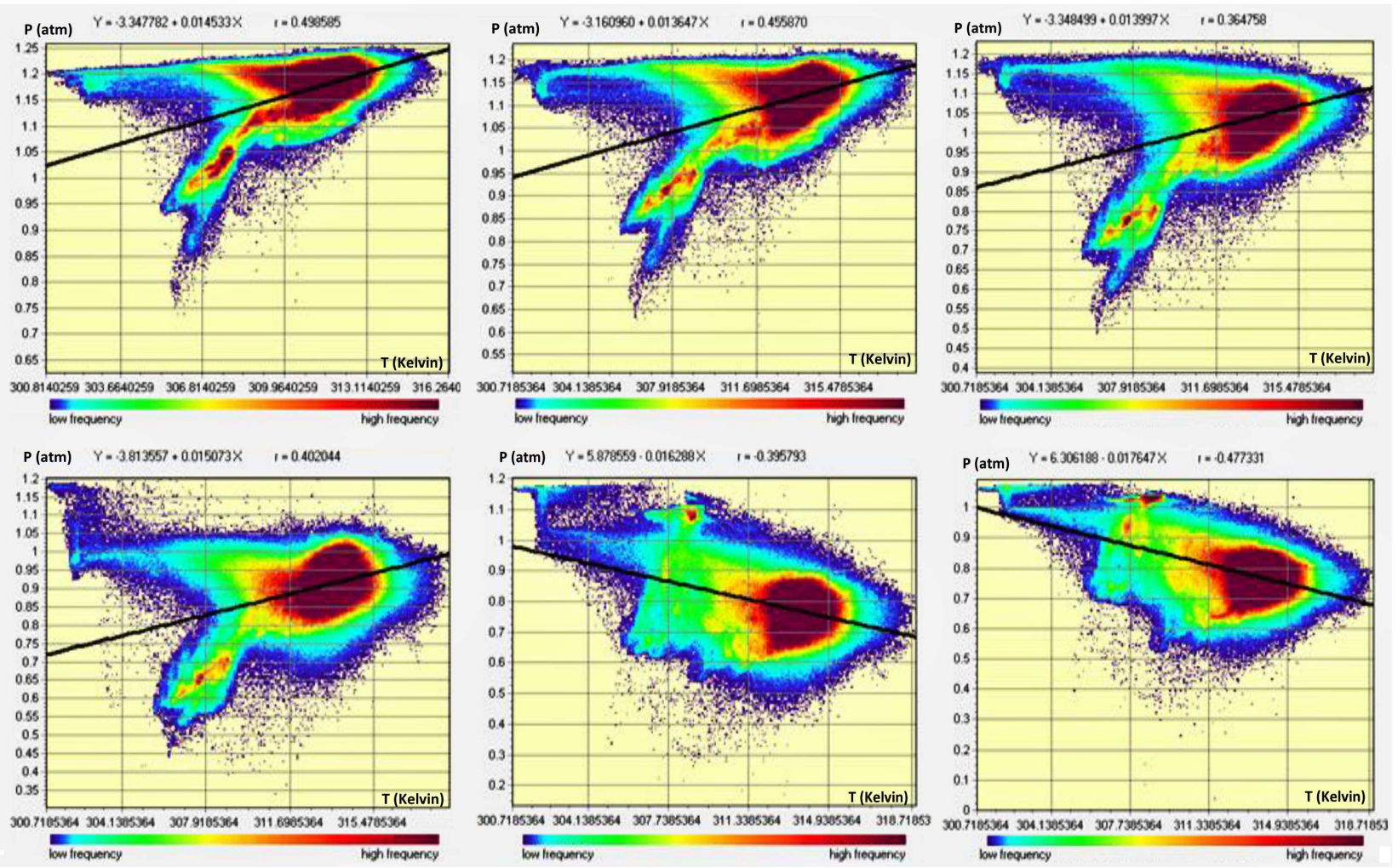

Fig. 2. Spectral scatter regression for osmotic pressure in Landsat 8 OLI (Band 2, 3, 4, 5, 6 and 7).

$$
\Pi=R T i M=C R T,
$$

where $\Pi$ is osmotic pressure in atmosphere (atm), $R$ is perfect gas constant $(R=8.314 \mathrm{~J} /(\mathrm{mol} \mathrm{K})), T$ is absolute temperature $(\mathrm{K})$, van 't Hoff factor is $i=2$ for strong electrolyte of $\mathrm{NaCl}, \mathrm{KCl}, C$ is the molar concentration ( $\mathrm{mol} / \mathrm{l}, \mathrm{g} / \mathrm{l}, \mathrm{g} / \mathrm{mol}$, osmoles/l) and $M$ is molarity or molality expressed as molar concentration $\left(\mathrm{mol} / \mathrm{m}^{2}\right)$.

The dissociation criteria in electrolyte concept is related to the wavelength of light path (sun position, viewing angle, solar constant, clouds, atmospheric parameter, etc.) and therefore to the solute type (molar concentration, volume, water temperature, dissociation percentage in each monochromatic wave band interacting within absorbance and transmittance of modeling salts).

\subsubsection{Top of atmospheric spectral radiance}

After inputting Band 10, in the background, Idrisi uses formulae taken from the USGS web page for retrieving the top of atmospheric (TOA) spectral radiance $L_{\lambda}$. The dark objects subtraction was applied to reduce low reflectance effect due to atmospheric Rayleigh scattering. This is necessary in order to use the Landsat 8 data [7]. This was done for all bands independently, after which all bands were stacked in one raster image in Idrisi. The conversion of DN to satellite brightness temperature (BT) for thermal bands can be found in [8]. The formula established for Landsat 8 for correction in Band $10(\mathrm{AL})$ is

$$
L_{\lambda}=\mathrm{ML} Q_{\text {cal }}+\mathrm{AL}
$$

where ML is a band-specific multiplicative rescaling factor from Landsat metadata, AL is a band-specific additive rescaling factor from Landsat metadata, $Q_{c a l}$ is a quantized and calibrated standard product pixel values (digital numbers, DN).

\subsection{Conversion of radiance to at-sensor temperature}

After the digital numbers (DNs) are converted to reflection, the thermal bands data are converted from spectral radiance to brightness temperature $T_{B}$ using the thermal constants provided in the Landsat 8 metadata file. The following equation is used to convert reflectance to BT [18]:

$$
\mathrm{BT}=K_{2} / \ln \left[\left(K_{1} / L_{\lambda}\right)+1\right],
$$

where $K_{1}$ is a constant $\left(\mathrm{W} /\left(\mathrm{m}^{2}\right.\right.$ sr $\left.\left.\mu \mathrm{m}\right)\right), K_{2}$ is a constant and $L_{\lambda}$ is the spectral radiance for Landsat 8 [8] at the sensor aperture measured in $\mathrm{W} /\left(\mathrm{m}^{2} \mathrm{sr} \mu \mathrm{m}\right)$.

The $K_{1}$ and $K_{2}$ constants for Landsat sensors are provided (for Landsat 8: image metafile, see Table I).

Constants of Landsat sensors series.

TABLE I

\begin{tabular}{c|c|c|c}
\hline \hline Const & Landsat $4^{a}$ & Landsat $^{a}$ & Landsat $7^{b}$ \\
\hline$K_{1}$ & 671.62 & 607.76 & 666.09 \\
$K_{2}$ & 1284.30 & 1260.56 & 1282.71 \\
\hline
\end{tabular}

${ }^{a}$ from Chandler and Markham (2003)

${ }^{b}$ from NASA (2011) 


\subsubsection{Emissivity correction by NDVI index}

There are several studies about the calculation of land surface temperature. For instance, using NDVI for the estimation of land surface emissivity [15], or using a land cover classification for the definition of the land surface emissivity of each class [19]. For instance, the emissivity $e$ values of various land cover types are provided from tables [9]. Multispectral bands were explored for calculating the normal difference vegetation index (NDVI). The importance of estimating the NDVI is essential and it can be used to infer general vegetation condition [11]. The calculation of the NDVI is important because, afterwards, the proportion of the vegetation $(p v)$ should be calculated, and they are highly related with the NDVI, and emissivity $e$ should be calculated from the formulae

$$
\operatorname{NDVI}=\frac{\operatorname{NIR}(\text { Band } 5)-\operatorname{Red}(\text { Band } 4)}{\operatorname{NIR}(\text { Band } 5)+\operatorname{Red}(\operatorname{Band} 4)},
$$

where NIR - near-infrared band (Band 5) and Red band (Band 4) of Landsat 8 OLI (operate land imager).

A method for calculating proportion of vegetation " $p v$ " [19] suggests using the NDVI values for vegetation and soil $\left(\mathrm{NDVI}_{v}=0.5\right.$ and $\left.\mathrm{NDVI}_{s}=0.2\right)$ to apply in standardized conditions is given by

$$
p v=\left(\frac{\mathrm{NDVI}-\mathrm{NDVI}_{\mathrm{S}}}{\mathrm{NDVI}_{\mathrm{V}}-\mathrm{NDVI}_{\mathrm{S}}}\right)^{2} .
$$

However, since the NDVI values differ for every area, the value for vegetated surfaces, 0.5 , may be too low. Global values from NDVI can be extracted from reflectivity, but it is impossible to establish global values in the case of an NDVI computed from TOA reflectivity, since it depends on the atmospheric conditions [14].

\subsubsection{Calculating land surface emissivity}

The land surface emissivity (LSE) is crucial in order to estimate LST, since the LSE is a proportionality factor that scales blackbody radiance (Planck's law) to predict emitted radiance, and it is the efficiency of transmitting thermal energy across the surface into the atmosphere [14]. The determination of the soil emissivity is calculated as suggested by

$$
\epsilon=\epsilon_{v} p v+\epsilon_{s}(1-p v)+C,
$$

where $\epsilon_{v}$ and $\epsilon_{s}$ are the vegetation and soil emissivity, respectively, and $C$ represents the surface roughness $(C=0$ for homogeneous and flat surfaces) which take in a constant value of 0.005 [15]. When the NDVI is less than 0 , it is classified as water, and the emissivity value of 0.991 is assigned. For NDVI values between 0 and 0.2 , it is considered that the land is covered with soil, and the emissivity value of 0.996 is assigned.

Values between 0.2 and 0.5 are considered mixtures of soil and vegetation cover and extracted to retrieve the mean emissivity. In the last case, when the NDVI value is greater than 0.5 , it is considered to be covered with vegetation, and the value of 0.973 is assigned. The last step of retrieving the LST or the emissivity-corrected land surface temperature is computed as follows:

$$
\mathrm{LST}=T_{B} /\left[1+\left(\lambda T_{B} / p\right) \ln (\mathrm{LSE})\right],
$$
where $\lambda$ is wavelength of emitted radiance, $p=h c / k_{\mathrm{B}}=$ $1.438 \times 10^{-2} \mathrm{~m} \mathrm{~K}, h=6.626 \times 10^{-34} \mathrm{~J} \mathrm{~s}$ is Planck's constant, $k_{\mathrm{B}}=1.38 \times 10^{-23} \mathrm{~J} / \mathrm{K}$ is the Boltzmann constant, $c=2.998 \times 10^{8} \mathrm{~m} / \mathrm{s}$ is velocity of light. The values of $\lambda$ for the Landsat thermal are listed in Table II.

TABLE II

Center wavelength $\lambda$ calibrated for thermal bands

\begin{tabular}{l|c|c}
\hline \hline \multicolumn{1}{c|}{ Satellites } & Bands & $\lambda[\mu \mathrm{m}]$ \\
\hline Landsat 4, 5, and 7 & 6 & 11.45 \\
Landsat 8 & 10 & 10.8 \\
Landsat 8 & 11 & 12
\end{tabular}

\section{Results}

In the software interface elaborated in $\mathrm{C}++$ language, we can choose parameters of experience which are the salt spectroscopy dataset (spectral reflectance of $\mathrm{NaCl}$ and $\mathrm{KCl}$ ) permitting extraction attributes such as the absorption coefficients in monochromatic rays, absorbance, transmittance, osmotic pressure related to temperature, and molar concentration of electrolyte.

\subsection{Calibration of spectroscopy} and Landsat OLI/TIRS wave bands

At low concentrations in good solvents, linear fitting obeys the van 't Hoff equation but not at higher concentrations. For all solvents at higher concentrations, this law should not be applied. We have adapted the multi regression fitting for physical and chemical parameters obtained automatically from spectroscopy database and spectral waveband of Landsat 8 sensor (from Table III to Table VI).

We have seen that osmotic pressure is influenced within temperature at SWIR domain (negative values) and is more sensitive for the blue and costal band, which is affected by aerosol scattering diffusion and relative humidity. We hope to differentiate clearly between osmotic and ionic effects by combining the spectral, spatial, and related physical parameters such as concentration, absorbance, transmittance, osmotic pressure, and temperature within Landsat sensor (Tables IV-VII). Hydrostatic pressure and low temperature act synergistically in molar concentration and increase in blue and coastal aerosols.

The osmotic pressure and molar concentration confirmed a linear relationship; and van 't Hoff equation at certain temperature is closed to the linear fit when molar concentration is less than $10 \mathrm{mg} / \mathrm{l}$. The best precision of concentration determination has an absorbance of 0.5 to 1 ( 10 to $60 \%$ transmittance) depending on the source of random noise. The concentration values increase for much dissociated solute such as $\mathrm{NaCl}$ salt for both blue band coastal aerosols band from 0.725 to $1.45 \mathrm{mg} / \mathrm{l}$. The proportionality to concentration means 
Regression fitting of osmotic pressure related to the brightness temperature in Landsat 8 .

TABLE III

\begin{tabular}{l|c|c|c}
\hline \hline \multicolumn{1}{c|}{ Spectral band } & $\begin{array}{c}\text { Regression fitting } \\
\text { of osmotic pressure }\end{array}$ & $\begin{array}{c}\text { Correlation } \\
\text { coefficient [\%] }\end{array}$ & $\begin{array}{c}\text { Spectra } \\
\text { intervales }\end{array}$ \\
\hline Band 1 - coastal aerosols & $P_{O}=-2.325262+0.01123 T$ & 57.2 & $0.43-0.45$ \\
Band 2 - blue & $P_{O}=-2.598082+0.01205 T$ & 52.6 & $0.45-0.51$ \\
Band 3 - green & $P_{O}=-3.16096+0.013647 T$ & 45.6 & $0.53-0.59$ \\
Band 4 - red & $P_{O}=-3.348499+0.01399 T$ & 36.5 & $0.64-0.67$ \\
Band 5 - near infra-red & $P_{O}=-3.813557+0.01628 T$ & 40.2 & $0.85-0.88$ \\
Band 6 - SWIR1 & $P_{O}=5.878559-0.017647 T$ & -40 & $1.57-1.65$ \\
Band 7 - SWIR2 & $P_{O}=6.306188-0.017647 T$ & -48 & $2.11-2.29$ \\
Band 8 - PAN & $P_{O}=-3.196537+0.01367 T$ & 41.5 & $0.5-0.68$ \\
Band 10 - TIRS 1 & $P_{O}=-2.598082+0.01205 T$ & 52.64 & $10.6-11.19$ \\
Band 11 - TIRS 2 & $P_{O}=-3.347782+0.01453 T$ & 50 & $11.50-12.51$
\end{tabular}

TABLE IV

TABLE VI

Best fitting regression between molar concentration of $\mathrm{NaCl}$ and absorbance in Landsat 8.

\begin{tabular}{c|l|c}
\hline \hline $\begin{array}{c}\text { Spectral } \\
\text { band }\end{array}$ & $\begin{array}{c}\text { Regression equation } \\
\text { of NaCl concentration }\end{array}$ & $\begin{array}{c}\text { Molar } \\
\text { concentration }\end{array}$ \\
\hline Band 1 & $C_{1}=0.69 A_{1}$ & $0.85-1.45$ \\
Band 2 & $C_{2}=0.69 A_{2}$ & $0.725-1.435$ \\
Band 3 & $C_{3}=0.69 A_{3}$ & $0.6-1.42$ \\
Band 4 & $C_{4}=0.69 A_{4}$ & $0.46-1.42$ \\
Band 5 & $C_{5}=0.69 A_{5}$ & $0.35-1.42$ \\
Band 6 & $C_{6}=0.69 A_{6}$ & $0.15-1.42$ \\
Band 7 & $C_{7}=0.69 A_{7}$ & $0-1.28$ \\
Band 8 & $C_{8}=0.69 A_{8}$ & $0.42-1.43$
\end{tabular}

TABLE V

Calibrated radiance and calibrated brightness temperature for given spectral band in Landsat 8

\begin{tabular}{c|c}
\hline $\begin{array}{c}\text { Spectral } \\
\text { band }\end{array}$ & \multicolumn{1}{|c}{$\begin{array}{c}\text { Regression fitting - radiance } \\
\text { and brightness temperature }\end{array}$} \\
\hline Band 10 & $\begin{array}{l}\text { Radiance } 10=-37.223323+0.155838 T_{10} \\
\text { and } T_{10}=238.872552+6.415824 \text { Radiance } 10\end{array}$ \\
Band 11 & $\begin{array}{l}\text { Radiance } 11=-30.024396+0.129748 T_{1} 1 \\
\text { and } T_{11}=231.412676+7.766507 \text { Radiance } 11\end{array}$
\end{tabular}

that osmotic pressure is a colligative property. In the absence of the semipermeable medium, diffusion would continue until the concentrations of all substances are uniform throughout the liquid phase. The experimental spectroscopy database USGS have been implemented by using Curve Expert software for best fitting models adaptation for each monochromatic ray accordingly within Landsat 8 OLI wavebands.

The results were obtained for analytical optical modeling by wavelength and related radiance, reflectance, and spectroscopy field of $\mathrm{NaCl}$, for example (Table VI). In the next table (Table VII), we observe that the short wavelengths properties are the most diffusing accordingly by sigmoidal model adaptation. A best correlation has been found by vapor pressure (exponential
Regression optical modeling using radiance and reflectance of $\mathrm{NaCl}$ spectroscopy database (Landsat 8).

\begin{tabular}{c|c}
\hline $\begin{array}{c}\text { Spectral } \\
\text { band }\end{array}$ & $\begin{array}{c}\text { Regression equation } \\
\text { of radiance and reflectance }\end{array}$ \\
\hline Band 2 & Radiance $2=45.531209+553.394107$ Reflectance 2 \\
Band 3 & Radiance $3=25.433714+509.946347$ Reflectance 3 \\
Band 4 & Radiance $4=13.185506+430.021997$ Reflectance 4 \\
Band 5 & Radiance $5=3.690037+263.153413$ Reflectance 5 \\
Band 6 & Radiance $6=-1.485158+65.441786$ Reflectance 6 \\
Band 7 & Radiance $7=-2.489891+22.058301$ Reflectance 7 \\
Band 8 & Radiance $8=20.446978+486.650213$ Reflectance 8
\end{tabular}

model) for the blue wave band (99.43\%) and the dose response model (DR-Hill) for panchromatic one (98.4\%). The results confirm that for small particles with wavelength below $10 \%$ of diameter, the Rayleigh diffusion depends on waveband, the concentration of electrolyte and the wavelength polarization. The diffusion decreases when wavelength increases, in the case of molecular spectrophotometry. We note that the spectral resolution increases from multispectral sensor to ultraspectral wavebands from $100 \mathrm{~nm}$ for Landsat 8 to $10 \mathrm{~nm}$ for hyperspectral sensor. The slope constant for OLI bands signify the monochromatic between concentration and absorbance in the $\mathrm{NaCl}$ electrolyte case (Table IV). This implies the relationship between optical thickness and mitigation absorption coefficients.

\subsection{Salinity detection and mapping from spectroscopy remote sensing technique}

In order to develop a consistent salinity modeling across all physical variables, the absorbance parameter is selected for each Landsat wavebands transformations. Finally, three consistent parameters are chosen as the radiative salinity modeling (osmotic pressure, concentration, and land surface temperature). Fitted regression models are tested for calibration processing between database spectroscopy and Landsat imageries according to the absorbance related to the monochromatic wavelength determined by the Beer-Lambert law 
TABLE VII

Best fitting regression models between wavelength and radiance of $\mathrm{NaCl}$ spectroscopy data using Landsat 8 .

\begin{tabular}{c|l|l|c|c}
\hline $\begin{array}{c}\text { Spectral } \\
\text { band }\end{array}$ & \multicolumn{1}{|c|}{ Regression equation } & Correlation coefficient & Radiance (r) [\%] & Models adaptation \\
\hline Band 2 & $\lambda=\exp (a+(b / x)+c \ln (r))$ & $\begin{array}{l}a=-227.39, b=13843.6, \\
c=32.0092\end{array}$ & 99.43 & vapor pressure (exponential) \\
\hline Band 3 & $\lambda=\alpha+\theta x\left(\theta /\left(\kappa^{\eta}+r \eta\right)\right.$ & $\begin{array}{l}\alpha=0.46, \theta=0.44, \\
\eta=45.52, \kappa=451.3\end{array}$ & 98.37 & dose response model (DR-Hill) \\
\hline Band 4 & $\lambda=a+b \cos (c r+d)$ & $\begin{array}{l}a=0.644, b=0.036, \\
c=0.407, d=-78.7022 \\
a=0.798, b=0.085, \\
c=0.536, d=-66.703\end{array}$ & 86.78 & sinusoidal \\
\hline Band 5 & $\lambda=a+b \cos (c r+d)$ & $\begin{array}{l}a=1.458, b=0.273, \\
c=3.734, d=341.526\end{array}$ & sinusoidal \\
\hline Band 6 & $\lambda=a+b \cos (c r+d)$ & $\begin{array}{l}a=2.864, b=-259.75, \\
c=-18.3, d=190.771\end{array}$ & sigmoidal - miscellaneous \\
\hline Band 7 & $\lambda=a /(1+\exp (b-c x))^{1 / d}$ & $\begin{array}{l}\alpha=0.47, \theta=0.43, \\
\eta=45.52, \kappa=451.3\end{array}$ & sigmoidal - model Richards \\
\hline Band 8 & $\lambda=\alpha+\theta r^{\theta / \kappa^{\eta}+r \eta}$ & 98.4 & dose response model (DR-Hill)
\end{tabular}

and the van 't Hoff law for ideal diluted solutions. Globally, there are several possibilities for spectral auto scaling using the best prediction model, which has the strongest correlation across five models of exponential vapor pressure, dose response, sigmoidal, sinusoidal, and linearity of Beer-Lambert, is retained.

The results confirmed that the best predictable fitted models are more suitable in waveband threshold: 260, 1400 , and $2100 \mathrm{~nm}$. In the literature, it is difficult to extract a specific spectral signal solely from only reflectance spectra [20]. Furthermore, we have performed the previous formulae relevant to the Beer-Lambert and van 't Hoff laws for the models with best calibration, for example, halite salt using the blue band of Landsat 8 under Idrisi software (Fig. 2). We note that osmotic pressure increases from blue to infrared wavebands and continues to decrease for short-wave wavelength. This is due to the water absorption that affects the solute polarizability on many minerals salts. Due to the high correlation between blue and panchromatic wavebands for halite salt, the absorbance related to the wavelength can be represented by the Lambertian absorption. The models demonstrate that the wavebands near 260, 1400, and $2100 \mathrm{~nm}$ are sensitive to humidity and salinity absorption attenuation spectra (Fig. 3).

In order to perform fitting modeling of $\mathrm{NaCl}$, we have used the curve expert software to extract spectral response and managed spectral attributes related to transmittance by fitting the best adapted discretized models in each waveband for Landsat 8 OLI/TIRS satellite implementation. We can detect the electronic and vibrational transitions directly in the molecular level at each waveband (Fig. 4).

There are two principal instrumental limitations to the Beer-Lambert law. The first limitation is that this law assumes that the radiation reaching the mineral is of a single wavelength when radiation is purely monochromatic. For this reason, it is better to make absorbance detection at the top of a broad absorption peak. In addition, the deviation from the Beer-Lambert is erroneous if the effective source bandwidth is less than onetenth of the natural bandwidth of the absorbing species. When measurements must be made on a slope, linearity is improved by using a narrower effective bandwidth. Stray radiation is the second contribution to instrumental deviations from Beer's law. Stray radiation arises from imperfections in the wavelength that allows light to enter the instrument and reach the detector without passing through the electrolyte. For a small concentration of analyte, transmittance is significantly smaller and the absorbance is unaffected by the stray radiation. At a higher concentration of analyte, however, less light passes through the analyte and transmittance may be similar in magnitude. This results in an absorbance that is smaller than expected, and a negative deviation from Beer's law. If we need to monitor an analyte concentration over time, it may be possible to manipulate automatically the tool providing optical analysis calibrated using the Beer-Lambert and van 't Hoff formalisms without any instrumental constraints.

A chemical deviation from Beer's law may occur if the analyte is involved in an equilibrium reaction. To hold $\alpha$ constant we buffer each standard to the same $\mathrm{pH}$.

Depending on the relative values of $\alpha_{\text {acid weak }}$ and $\alpha_{\text {analyte, the calibration curve has a positive or a neg- }}$ ative deviation from the Beer-Lambert law if we do not buffer the standards to the same $\mathrm{pH}$ at the same wavelength. The equation as described as

$$
C_{a}=\left(1-\alpha_{\text {acid weak }}\right) C_{\text {total }},
$$

where $C_{\text {total }}$ is weak acid's total concentration, $C_{a}$ are the equilibrium concentrations, $\alpha_{\text {acid weak }}$ are activity coefficients of analyte solution. 

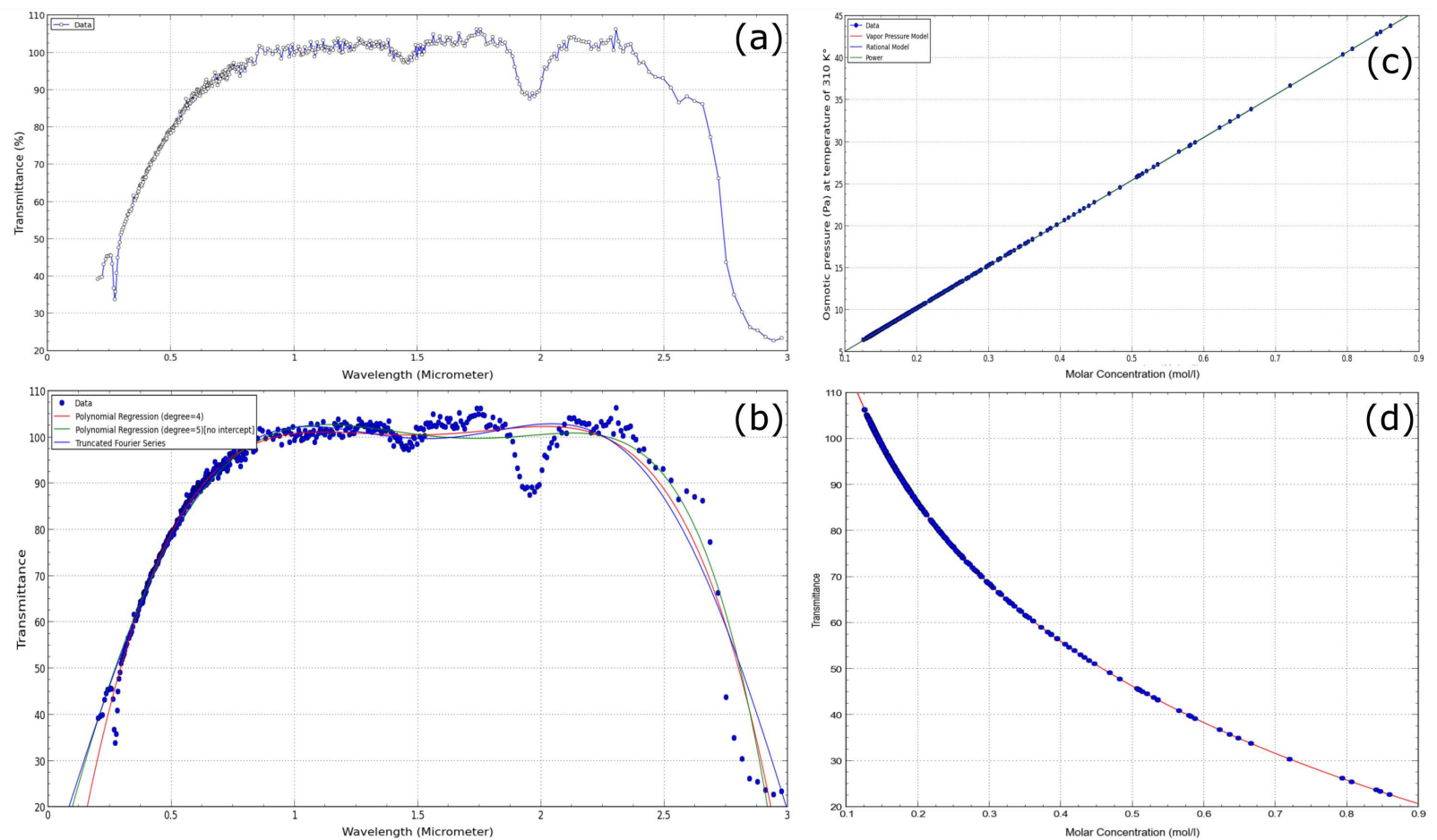

Fig. 3. (a) Transmittance of the Halite salt NaCl, (b) Polynomial fitting of NaCl Transmittance, (c) Linearity between osmotic pression and molar concentration for $\mathrm{NaCl}$, and (d) Exponential model fitting for NaCl between molar concentration and transmittance.
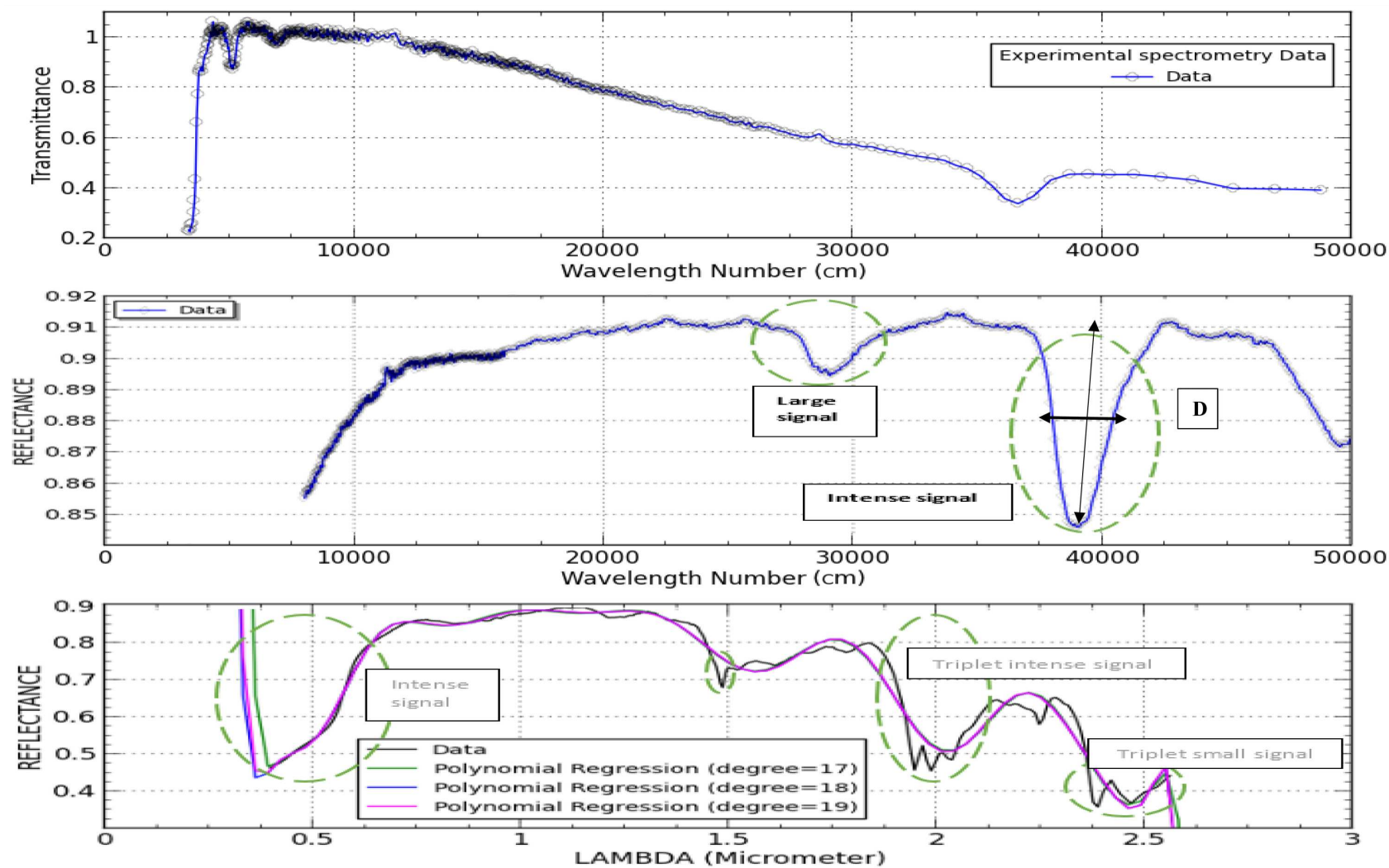

Fig. 4. Spectral response of Halite salt within transmittance, reflectance for $\mathrm{NaCl}$ and polyhalite (at last position) by electronical and vibrational transitions for experimental data fitted under Curve Expert software. 
When light passes through the compound, energy from the light is used to promote an electron from a bonding or non-bonding orbital into one of the empty anti-bonding orbitals (Fig. 5). Only a limited number of the possible electron that jumps absorb light in that region.

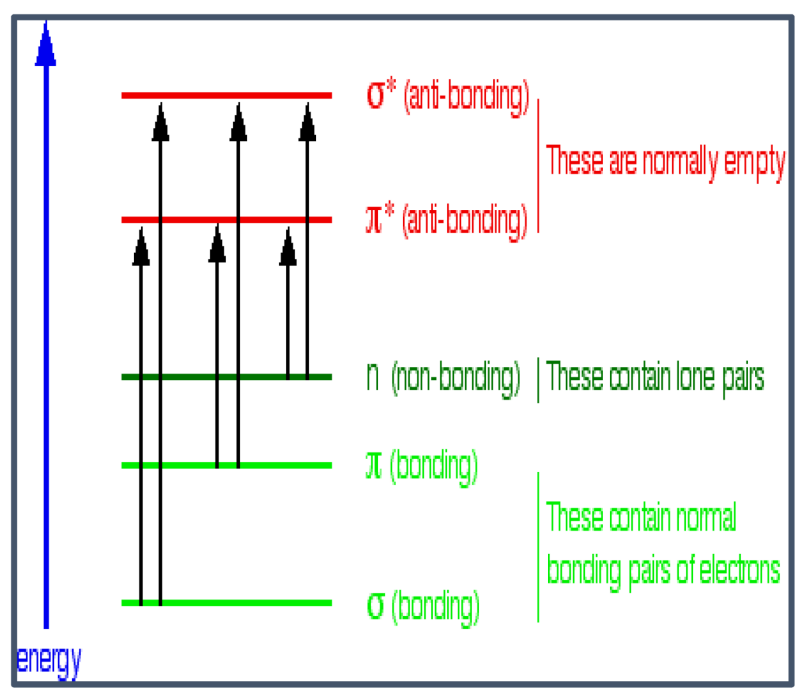

Fig. 5. Energetic diagram for molecular spectroscopy.

The non-bonding orbital has a higher energy than a $\pi$ bonding orbital. That means that the jump from an $\mathrm{NaCl}$ lone pair into a $\pi$ anti-bonding orbital needs less energy. That means it absorbs light of a lower frequency and therefore, of a higher wavelength. $\mathrm{NaCl}$ can therefore absorb light of two different wavelengths:

- The $\pi$ bonding to $\pi$ anti-bonding absorption peaks at $1458 \mathrm{~nm}$;

- The non-bonding to $\pi$ anti-bonding absorption peaks at $1950 \mathrm{~nm}$.

The decrease in power of monochromatic reflectance passing through a homogeneous absorbing medium is stated quantitatively by the Beer-Lambert law.

Finally, a calibration curve is constructed to determine the range of concentrations for which Beer's law is valid. Additional considerations that are important in any quantitative method are the effects of potential interferences.

The relation between osmotic pressure and molar concentration has in small part shape of a linear curve due to the equality of osmotic pressure between two sides of the salt medium.

Therefore, it can only be adapted to the dilute solution by optical transmissivity inversion fitting. Nonlinear effect is observed within wavelength of $\mathrm{NaCl}$ spectra and seems to be fitted to the exponential law until the path length of one meter (light diffraction and molecular diffusion effect) and continue to increase to flat linearity because the Fresnel reflexion coefficients affect the ray diffraction at the grain-solute contact (Fig. 6). We illustrate that spectral curve of halite and sylvite salts have more intense electronic and vibrational effects in the shortwave field according their solubility product, with other chromophores, such as water, due to the presence of salt. The electronic excitation leads to transitions from $\pi$ to $\pi^{*}$. However, it is even easier to excite nonbinding electrons to higher energy states. Absorption peaks manifest these transitions.

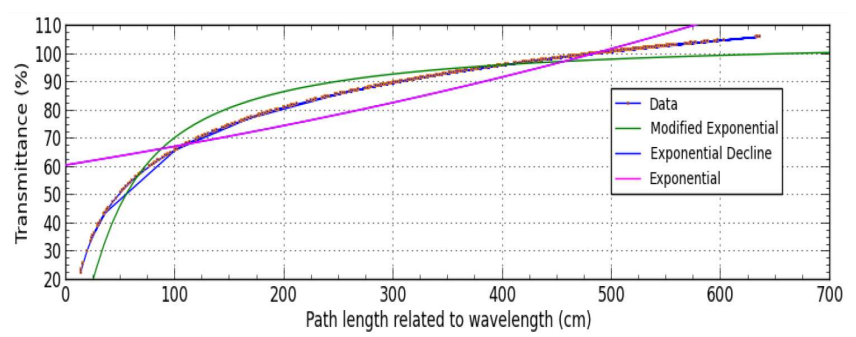

Fig. 6. Exponential fitting of $\mathrm{NaCl}$ transmittance at path length related to the wavelength curve.

A transmittance of $100 \%$ means no absorption. Therefore in infrared waveband, the absorption deflects downwards by curve spectroscopy determination.

In order to validate both Beer-Lambert and van 't Hoff laws accordingly using remote sensing spectrometry of salts such as $\mathrm{NaCl}$ (halite) and $\mathrm{KCl}$ (sylvite), the new tool have been established to discriminate salt spectra attributes by detection and identification through the Landsat sensors (Fig. 7). As we see in the snapshot below, a constant path length differs for all incident photons from Landsat 8 sensor. This means an unequal absorber concentration across the light insure homogeneity and changes has been made in reflectance properties at high concentration because most applications obey to the linear fitting at lower concentrations.

The absorption spectrum is flat over the spectral waveband by using Beer-Lambert by monochromatic rays. This implies that the Lambertian photometry conduct the same absorption coefficients in target, which match with Beer's law applicability. The concentration error is very small and negligible compared to other ones. However, in situ absorption according inhomogeneous liquid seems to be imperfect.

To observe a significant absorption in infrared domain, it is necessary to vary the dipole moment of the molecule during the vibration. The spectral salinity variation is referred to as the absorbance symmetrical connections.

The groups of atoms which absorb are called chromophore groups and those which do not absorb but only cause changes in absorption by a chromophore are called auxochromes. When a chromophore is subjected to electronic influences, the absorption band can move towards the strong wavelengths, which is the bathochromic effect, or towards the weak wavelengths, which is the hypochromic effect. If the light absorption is increased, a hypochromic effect is due. 


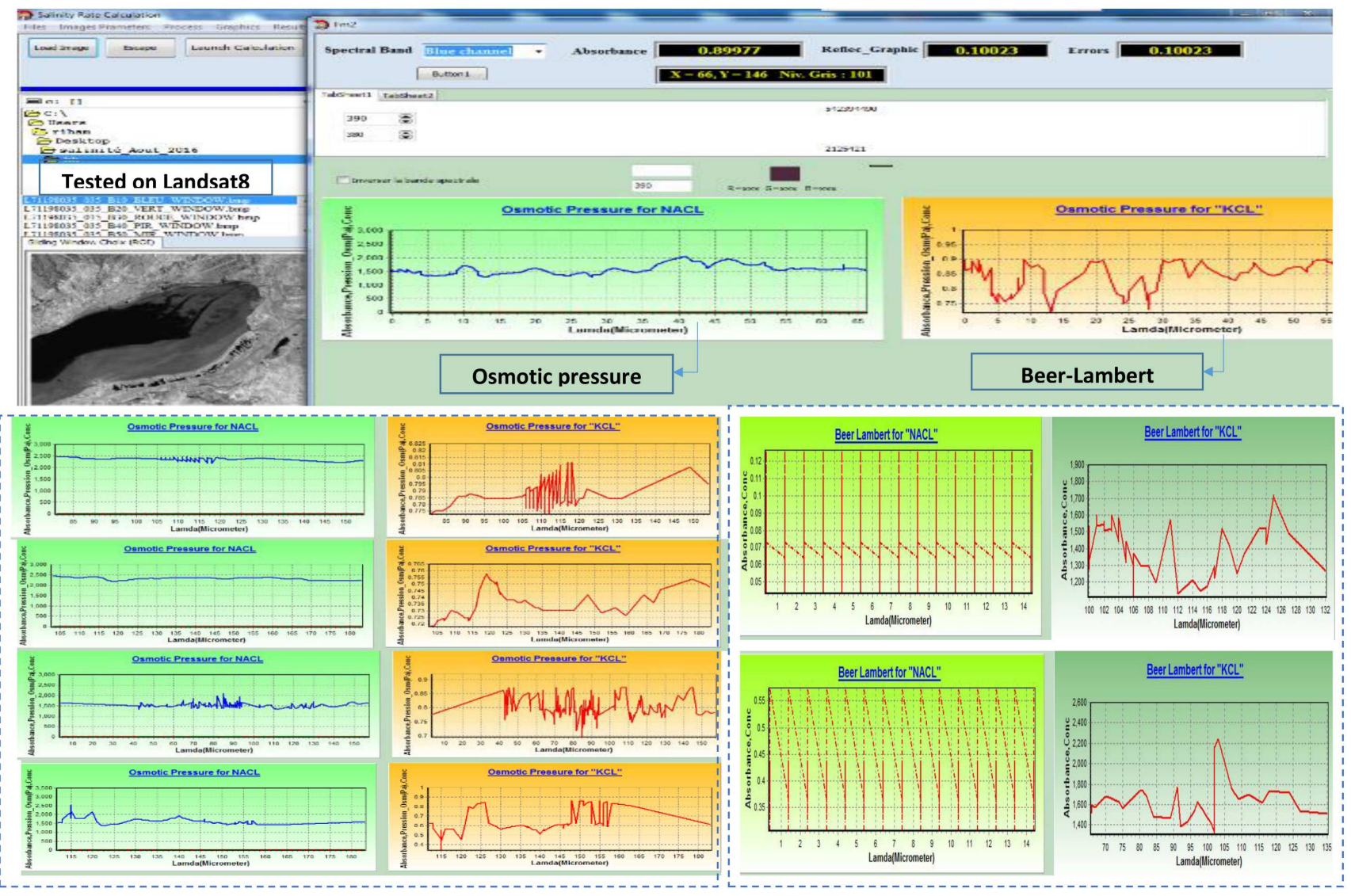

Fig. 7. New tool software for spectroscopy remote sensing showing the absorbance of both $\mathrm{NaCl}$ and $\mathrm{KCl}$ salts related by osmotic pressure (van 't Hoff law) in blue, green, red and short-infrared (left side) and Beer-Lambert law for just blue and near infrared (right side).

\section{Conclusion}

The purpose of this paper was to develop an optical remote sensing technique for detection and identification of spectral salts such as the solubility solutions of $\mathrm{NaCl}$, $\mathrm{KCl}$ using the spectroscopy concept under the formalism of the Beer-Lambert and van 't Hoff laws.

Furthermore, the new spectral approach is more adaptive for extracting related physical and chemical parameters automatically in the Landsat 8 OLI/TIRS imagery for dilute solutions. Whereas, a validation within experimental spectroscopy database was implemented both for fitting adaptation models and linearity calibration for suitable corrected reflection of $\mathrm{NaCl}$ and $\mathrm{KCl}$ under ideal conditions of applicability.

Oriented solutions have taken in consideration the polychromatic light effect which is mentioned under Landsat $8 \mathrm{OLI} /$ TIRS that affects the linearity calibration by noise effect for scattered reflectance. Increase in the maximum absorptivity to three implies that the linearity is weak because of stray light effect, which influences the absorbance above two. The best precision of concentration calculation is reached for values of absorbance between 0.5 and 1 (10 to $60 \%$ transmittance) due to the dominant source of random noise in Landsat sensor.
The linearity concept by spectroscopy remote sensing is actually improved due to existence of two effects, stray and polychromatic light. The first one is stronger if the wavelength increases.

Furthermore, the applicability of Beer-Lambert imposes limitless due to the peak waveband for weak concentration and the alternative wavelength for higher concentration.

The image processing is substantially inadequate if absorption increases considerably above 3 within a mediumresolution of Landsat 8 . In that case, the spectral interval might be larger than the absorption width, and makes the spectral shape set to Lorentzian concept. The BeerLambert law demonstrates that all the photons striking the Landsat 8 detector infer to the same absorption coefficient (homogeneous shape) by the analyte like $\mathrm{NaCl}$ and $\mathrm{KCl}$.

Results confirmed that osmotic pressure and the BeerLambert law are the best conducted approach for salinity modeling using remote sensing spectroscopy. The values obtained by regression fitting in experimental modeling are more sensitive to the satellite instrumentation board and atmospheric conditions that interfere due to reflectance and absorbance in electrolyte solution. 
In case of non-linearity of Lambert's law curve, the effect is optical rather than chemical. The spectral curve is linear at low concentrations but non-linear at high concentrations, essentially due to stray light. In different case, Beer-Lambert law curve shape is in part and nonlinear in other one, which implies that the non-linearity is chemical in nature. Osmotic pressure is adapted exclusively by dilute solutions when the concentration difference is very small.

The tool addressed for this paper is most important for detection and identification of salts taking in account the energetic vibrational and electronic transitions for salt molecule absorption in shortwave field exceptionally and permitted to overcome the limit experimentation about both Beer-Lambert and van 't Hoff laws.

The results confirmed that for small particles below $10 \%$ of diameter at nanometric wavelength, the Rayleigh diffusion predominates depending on waveband, the electrolyte concentration, and the wavelength polarization. The diffusion decreases when wavelength increases, which remains the case of molecular spectroscopy.

The results confirm that the short wavelength properties are the most diffusing accordingly by sigmoidal model fitting. However, the best correlation has been identified by vapor pressure (exponential model) for the blue waveband $(99.43 \%)$ and the dose response model (DR-Hill) for panchromatic band (98.4\%).

In the future, we advise to enlarge knowledge of our optical detection with routine applications inspired from analytical chemistry by using more versatile spectrophotometer to adjust experimental and instrumental conditions. The optical properties of $\mathrm{NaCl}$ sample may change significantly with wavelength due to contamination and degradation. We can minimize this problem by measuring absorbance relative to a baseline established for the absorption band.

For solid biomaterials especially in the chemometric analysis the corrected Fourier transform in infrared spectra is used based on extinction coefficient matrix in pure water spectrum (water is active at $2100 \mathrm{~cm}^{-1}$ ) which represents a required reference band for the subtraction compared to the solid biomaterial in order to eliminate the interference from water molecules absorbed.

The tool established exprime validity of the sensitivity of a molecular absorption method, selectivity for multi component analysis, relatively attainable accuracy at $10 \%$ if spectral and chemical interferences are minimized, and finally quick integration computer simulation, under dissociation of analyte into free atoms.

In the future, we suggest using spectroscopic transducers such as thermal and photon transducers. They are promoted for development in spectroscopy related to wide wavelengths: 0.8 to $40 \mu \mathrm{m}$ for thermistor (change in resistance output signal), 0.8 to $1000 \mu \mathrm{m}$ for pneumatic (membrane displacement), 0.3 to $1000 \mu \mathrm{m}$ for pyroelectric (current), 750 to $6000 \mathrm{~nm}$ for photoconductor (change in resistance: photon).

\section{Acknowledgments}

The authors are grateful to Center of Spatial Techniques CTS — ASAL Algerian Spatial Agency and to the LAAR Laboratory and Energetic Physics department from USTO-MB Oran. All Landsat 8 images has been downloaded from USGS website and database profile mentioned in this manuscript speclab.cr.usgs.gov/spectral.lib06/ds231/datatable.html. We would like to thank all anonymous reviewers for their valuable feedback that helped to improve this manuscript greatly.

\section{References}

[1] S.H. Gordon, A. Mohamed, R.E. Harry O Kuru, S.H. Imam, Appl. Spectrosc. 64, 448 (2010).

[2] O. Içelli, Z. Yalçin, V. Karakaya, AIP Conf. Proc. 1611, 199 (2014).

[3] R.N. Clark, G.A. Swayze, A. Gallagher, U.S. Geol. Survey Office Miner. Resour. Bull. 2039, 141 (1993).

[4] B. Mougenot, M. Pouget, G.F. Epema, Remote Sens. Rev. 7, 241 (1993).

[5] F. Becker, Z.L. Li, Int. J. Remote Sens. 11, 369 (1990).

[6] R.N. Clark, G.A. Swayze, R. Wise, E. Livo, T. Hoefen, R. Kokaly, S.J. Sutley, USGS Digital Spectral Library splib06a, U.S. Geological Survey, 2007, p. 231.

[7] S. Gilmore, A. Saleem, A. Dewan, CEUR Workshop Proc. 1323, 100 (2015).

[8] USGS database.

[9] F.M. Howari, P.C. Goodell, S. Miyamoto, J. Environm. Qual 31, 1453 (2002).

[10] G.I. Metternicht, J.A. Zinck, Remote Sens. Environm. 85, 1 (2003).

[11] Q.H. Weng, D.S. Lu, J. Schubring, Remote Sens. Environm. 89, 483 (2004).

[12] F. Wang, Z. Qin, C. Song, L. Tu, A. Karnieli, S. Zhao, Remote Sens. (7), 4268 (2015).

[13] J.A. Sobrino, J.C. Jiménez-Muñoz, L. Paolini, Remote Sens. Environm. 90, 434 (2004).

[14] J.C. Jiménez-Muñoz, J.A. Sobrino, A. Plaza, L. Guanter, J. Moreno, P. Martínez, Sensors 9, 768 (2009).

[15] C. Song, C.E. Woodcock, K.C. Seto, M.P. Lenney, S.A. Macomber, Remote Sens. Environm. 75, 230 (2001).

[16] P.S. Chavez Jr, Remote Sens. Environm. 24, 459 (1988).

[17] J. Mallick, C.K. Singh, S. Shashtri, A. Rahman, S. Mukherjee, Int. J. Appl. Earth Observ. Geoinform. 19, 348 (2012).

[18] NASA, Landsat 8 bands, 2016 and USGS, EarthExplorer, 2016.

[19] Inbar Y. Ben-Dor, Y. Chen, Remote Sens. Environm. 61, (1997).

[20] E. Ben-Dor, S. Chabrillat, J.A.M. Demattê, G.R. Taylor, J. Hill, M.L. Whiting, S. Sommer, Remote Sens. Environm. 113, S38 (2009). 